\title{
Case Report on Acupuncture Treating Wilson Disease
}

\author{
Zheng $\mathrm{Yu}^{1}$, Lu Luo ${ }^{2}$, Ziwen Wang ${ }^{3}$ and Fanrong Liang ${ }^{3 *}$ \\ ${ }^{1}$ College of Medical Information and Engineering, Chengdu University of TCM, Sichuan Province, China \\ ${ }^{2}$ Institute of Chinese Material Medical, China Academy of Chinese Medical Sciences, Beijing, China \\ ${ }^{3}$ College of Acumox and Tuina, Chengdu University of TCM, Sichuan Province, China
}

*Corresponding author: Fanrong Liang, College of Acumox and Tuina, Chengdu University of TCM, Sichuan Province, China.

Received Date: Decembe 16, 2020

Published Date: February 16, 2021

\begin{abstract}
Wilson disease (WD), an autosomal recessive disease with abnormal copper metabolism, is caused by mutations in the ATP7B gene. It is characterized by liver, nervous system, and ocular manifestations related to the deposition of copper in the liver, lens nucleus, and cornea. The case reported in this article was a 30-year-old woman with high muscle tension, unable to stand or walk, low voice, and short-term memory loss. Brain MRI showed patchy T1, long T2 signals, and high FLAIR showed signals in the basal ganglia thalamus area, subcortex, and brain stem. Low signal shadows were presented in magnetically sensitive bilateral basal ganglia region, callus, and cerebellar dentate nucleus. Ceruloplasmin was significantly reduced. With conventional treatment by western medicine, the symptoms were not alleviated. However, after the application of acupuncture-assisted treatment, the patient is capable with self-care ability, thus a desired effect was achieved. It is therefore suggested that acupuncture shall be applied to assist treatment of WD to relieve symptoms and help patients return to the society.
\end{abstract}

Keywords: Wilson disease; Hepatolenticular degeneration; Acupuncture

\section{Introduction}

Wilson disease (WD), also called Hepatolenticular Degeneration (HLD), is an autosomal recessive disease with abnormal copper metabolism, caused by mutations in the ATP7B gene [1]. ATP7B gene, which encodes a transmembrane copper-transporting ATPase, leading to copper overload in the liver, brain and other organs [2]. Copper is a crucial micronutrient needed by animals and humans for proper organ function and metabolic processes such as hemoglobin synthesis, as a neurotransmitter, for iron oxidation, cellular respiration, and antioxidant defense peptide amination, and in the formation of pigments and connective tissue [3]. Impaired ATP7B function in Wilson disease results in excessive accumulation of copper in liver, brain, and other tissues. Toxic copper deposits may induce oxidative stress, modify expression of genes, directly inhibit proteins, and impair mitochondrial function, leading to hepatic, neuropsychiatric, renal, musculoskeletal, and other symptoms [4].

WD usually occurs in childhood or adolescence, and it starts with hepatic and neurological manifestations. There are also a few patients whose first symptoms are neurological symptoms, hemolytic anemia, or renal impairment. But eventually, liver and nerve damage will appear. Most patients will have a K-F ring in the eye, but there are a few patients who don't [5-7]. Given that the occurrence rate is relatively low, clinicians lack experience in diagnosis. Therefore, it is common for patients without K-F ring manifestations fail to receive timely diagnosis and treatment, which always leads to organ malfunctions. A series of clinical symptoms 
are presented due to copper excretion disorders which leads to accumulation in human body, mainly including symptoms of the liver, nervous system and eyes, as well as the kidneys, cardiovascular system, heart, etc. The incidence of WD in China is higher than that in the west [8].

\section{Case Report}

A 30-year-old female patient paid her first visit to acupuncture clinic since she couldn't stand or walk. Her family complained that she suffered from hepatolenticular degeneration and the first diagnosis was made in 2007. However, in 2005, she developed jaundice, liver coma, and abnormal blood coagulation, yet no clear cause was found. She was discharged after the symptoms were relieved by western medical treatment. In 2007, she was diagnosed with nodular cirrhosis during gallstone surgery. Therefore, WD was diagnosed, followed by test showing significantly reduced ceruloplasmins. Additionally, copper drainage was given, and the patient was discharged after cholecystectomy. The patient continued to have hypoproteinemia, and long-term use of albumin. The hypoproteinemia was relieved after she was treated with acupuncture and Chinese herbal medicine for four months. In 2008, he had colectomy due to dolichosigmoid, the postoperative patient was in good physical condition and could take care of herself and be able to work normally. Until 2017, an unexplained diarrhea occurred, and her weight was reduced to $28 \mathrm{~kg}$. Then she had mental symptoms including visual hallucinations, fear, and speech rambling, etc. She couldn't walk stability, and cerebral hemorrhage occurred after falling down. She was then admitted to the Department of Neurology, West China Hospital for treatment.

The admission examinations showed that ceruloplasmin was $34.2 \mathrm{mg} / \mathrm{l}$, 24-hour urine copper was $0.55 \mathrm{umol} / \mathrm{l}$, prothrombin time was $13.2 \mathrm{~s}$, INR was 1.21 , D-dimer was $1.33 \mathrm{mg} / \mathrm{l}$. MRI of head showed that there were slightly longer T1 and T2 signals in bilateral basal ganglia thalamus, subcortical and brainstem, and higher flair signal. Low signal shadow was seen in the basal ganglia, substantia nigra and dentate nucleus of cerebellum. The ventricles, cisterns and sulci were widened, especially around the cerebellum. Midline structure was centralized. No abnormal signal is found in skull. ECG: sinus tachycardia, T wave changes. No abnormality was found in liver and kidney function and electrolyte. The cerebrospinal fluid was normal and TB antibody was negative; No abnormality was found in glucose-6-phosphate dehydrogenase 1131iu / L and anticardiolipin antibody Ru / ml; K-f ring was never found in the patient. The patient didn't follow low-cooper diet since the diagnosis till the inpatient admission. She was given quetiapine fumarate tablets, dobutazide tablets, escitalopram oxalate tablets and dimercapto butanediol tablets after admission. After 21 days of treatment, the patient was discharged from the hospital and could communicate with his family, but she was unable to stand or walk, and had difficulty in articulation. She again looked for acupuncture treatment. Since May 2019, she received acupuncture treatment in Prof. Liang Fanrong's clinic, three times per week. The acupoints were: GB 20 (Fengchi), GB 12 (Wan Gu), BL 10 (Tian Zhu), GB 8 (Shuai Gu), Du 20 (Baihui), EX-HN 1 (Sishengcong), EX-B 2 (Jiaji), PC 6 (Neiguan), SI 4 (Wangu), SP 9 (Yinlingquan), GB 34 (Yanglingquan), ST 36 (Zusanli), SP 6 (Sanyinjiao), KI 3 (Taixi), BL 60 (Kunlun), KI 2 (Rangu). The compatibility of acupoints shows in Table 1. Moxibustion box was applied on the acupoints at the back. After 14 times of treatments, the patient could walk for two meters without any assistance. Her menstruation also returned normal after a few years of pause. Until January 2020, she has totally received 50 times of treatments. Except for the low voice, the patient can take care of herself, and her short memory was returned as well. Both her family and her were satisfied with the effect.

Table 1: She is now continuing her acupuncture treatment in the clinic.

\begin{tabular}{|c|c|c|}
\hline Acupoints & Function & Compatibility \\
\hline Du 20 (Baihui) & Open the orifices and consolidate & \multirow{4}{*}{$\begin{array}{l}\text { Monarch (Jun) points, combined } \\
\text { together to open orifices, improve } \\
\text { cognition impairment and motor } \\
\text { disfunctions }\end{array}$} \\
\hline EX-HN 1 (Sishengcong) & Benefit the brain and calm down the mind & \\
\hline GB 20 (Fengchi) & Refresh the spirit and sedate liver wind & \\
\hline PC 6 (Neiguan) & Calm down the spirit, regulate $Q \mathrm{Q}$, and relieve pain & \\
\hline EX-B 2 (Jiaji) & Regulate organ functions & \multirow{6}{*}{$\begin{array}{l}\text { Minister (Chen) points, Benefit organs } \\
\text { and reinforce Qi and blood }\end{array}$} \\
\hline SP 9 (Yinlingquan) & Strengthen spleen and remove dampness & \\
\hline SP 6 (Sanyinjiao) & Strengthen spleen, tonify kidney and liver & \\
\hline ST 36 (Zusanli) & Tonify stomach qi and resolve spleen dampness & \\
\hline KI 3 (Taixi) & Nourish kidney yin and tonify kidney yang & \\
\hline BL 10 (Tian Zhu) & Activate Qi and reinforce Yang & \\
\hline GB 34 (Yanglingquan) & The tendon confluent point of eight confluent points & \multirow{3}{*}{$\begin{array}{c}\text { Assistant (Zuo) points, sommthe } \\
\text { meridians and relieve contractures of } \\
\text { tendons }\end{array}$} \\
\hline SI 4 (Wangu) & activate the meridians and remove dampness & \\
\hline BL 60 (Kunlun) & Clear the heat and activate the meridians & \\
\hline GB 12 (Wan Gu) & Clear the wind heat and improve eyesight & \multirow{2}{*}{$\begin{array}{l}\text { Assistant (Zuo) points, clear internal } \\
\text { wind, external heat and dampness }\end{array}$} \\
\hline KI 2 (Rangu) & Raise the clear and reduce the turbid & \\
\hline
\end{tabular}




\section{Discussion}

Early diagnosis is crucial for WD. Preferably when the child/ adolescent is asymptomatic, it is most likely to result in nearnormal longevity with generally good health so long as the patient tolerates effective medication, is adherent to the lifelong treatment regimen, and has consistent access to the medication [9]. An early diagnosis of WD, and appropriate anticopper treatment, usually leads to a marked improvement in patient health. Furthermore, if the treatment is performed before tissue damage, tissue damage can be avoided. Tissue damage can be reversed in most patients [10]. Conversely, delayed diagnosis can result in persistent pathology, which, left untreated, can ultimately prove lethal [11].

The clinical manifestations of WD are very complex, because copper accumulates not only in the liver and brain, but also in other organs. Liver injury can also be the cause of secondary impairment of other tissues. Therefore, the clinical manifestation of WD may involve liver, renal, cardiac, skin, osteoarticular, or endocrinologic and include other organ disturbances [12]. In almost $20 \%$ of cases, psychiatric disorders is the first clinical manifestation of WD [13]. Together with a combination of symptoms, it can easily lead to misdiagnosis.

For conventional medical treatment, copper chelating agents and zinc preparations were mostly applied. If early diagnosis and timely treatment are available, patients are expected to obtain a healthy life. However, all currently available WD treatments are associated with adverse effects in a subset of patients, such as neurological deterioration.

The patient in this article started with hepatic symptoms, unfortunately the diagnosis was not confirmed in time. The diagnosis of WD and corresponding treatments were given 2 years later, so that the patient could work and live normally in the next ten years. However, since she didn't strictly control the diet and receive treatment on time, neurological and psychiatric symptoms appeared. Through acupuncture-assisted treatment, the relatively ideal effect was achieved.

Traditional Chinese Medicine (TCM) hasn't made a clear statement regarding WD. Based on the symptoms of high muscle tension, unable to stand or walk, pale and thin tongue, deep and thin pulse, the TCM diagnosis was "Tendon Disease (Jing Bing)". The location of Tendon Disease is, apparently, at the tendons. Liver is in charge of tendons. According to the theory of five elements, kidney (corresponding to water) can nourish the liver (corresponding to wood), and liver restrains spleen (corresponding to earth). If liver is sick, it would over-restrain spleen, lead to spleen deficiency. According to Discussion on Tendons and Bones, Su Wen, Inner Canon of the Yellow Emperor (Huang Di Nei Jing), the disorders of Du vessels are manifested with contracture and intensity of neck and muscles. The collateral of Du vessel is connected with Shaoyin meridian, passing by the spin and belonging to the kidney. Therefore, WD, in TCM, is correlated to liver, spleen (stomach), kidney, and Du vessels. The contracture is caused by external blockage on the meridians, Qi and blood accumulation. As per transformations of the syndrome differentiation, it could turn into heat and fire flaming, which consumes the yin fluid; If it lasts for a long period or if the treatment is delayed, the liver essence and kidney blood shall be insufficient; If the symptoms include poor appetite and fatigue, then the spleen earth is deficient; If the chronic pathogens dwells into the collaterals, or there is external trauma, there will be blood stagnation and blocked vessels. Technically speaking, there could be deficient or excessive syndromes, contracture due to insufficient nutrition in the tendons and meridians, and this is the pathogenesis of WD in TCM diagnosis. While for this patient particularly, given her course of treatment, tongue and pulse, her syndrome differentiation was liver, spleen and kidney deficiency, combined with Qi deficiency and blood stasis. So acupoints Du 20 (Baihui), EX-HN 1 (Sishengcong), GB 12 (Wan Gu), SI 4 (Wangu), PC 6 (Neiguan), GB 20 (Fengchi), and BL 10 (Tian Zhu) were selected to relieve insomnia, depression, visual hallucinations and other mental symptoms. EX-B 2 (Jiaji) was chosed to regulate the zang and fu organs. Combining with moxibustion, it can strengthen the beneficial effect on functions of zang-fu organs. Puncturing SP 9 (Yinlingquan), together with GB 34 (Yanglingquan), extrapyramidal symptoms such as tremor, salivation, and high muscle tension were relieved. ST 36 (Zusanli) and SP 6 (Sanyinjiao) can reinforce the postnatal Qi, while KI 3 (Taixi), BL 60 (Kunlun), and KI 2 (Rangu) can tonify the prenatal Qi, in return to strengthen the postnatal Qi, so the blood could be transformed from the Qi. Through the combination of all the acupoints above, both Yin and Yang were reinforced, and Qi and blood were simultaneously regulated.

There are few reports on acupuncture treatment for WD, but several researches have shown that acupuncture could promotes neurogenesis and cell proliferation in the central nervous system [14] Additionally, acupuncture presents antagonizing oxygen stress and anti-free radical effect [15]. Meanwhile, omics' studies have found that acupuncture can regulate metabolism, recover the disordered functions, which might be the effective mechanism of acupuncture.

\section{Conclusion}

"To treat the same disease with different approaches and different diseases with the same approach", proposed by the basic theory of TCM, applies the same therapy for different diseases with the same syndrome pattern, while different approaches for the same disease with different patterns. Though the cause of WD is cooper accumulation, the pathogenesis is disordered functions of functions in TCM diagnosis. Therefore, acupuncture is trying to regulate the functions of organs thus to improve the physical state of body, such as the recovery of motor function and short memory, appetite, and menstrual cycle. In the process of WD treatment, acupuncture shall be applied to relieve symptoms, which is 
conducive to help the patients return to society, and in consequence reduce the medical and social burden. The concept of "to treat the same disease with different approaches and different diseases with the same approach" could be applied in various disorders as well.

\section{Acknowledgement}

None.

\section{Conflict of Interest}

Author declares no conflict interest.

\section{References}

1. Schilsky ML (2017) Wilson Disease: Diagnosis, Treatment, and Follow-up. Clin Liver Dis 21(4): 755-767.

2. Członkowska A, Litwin T, Dusek P, Ferenci P, Lutsenko S, et al. (2018) Wilson disease. Nat Rev Dis Primers 4(1): 21.

3. Myint ZW, Oo TH, Thein KZ, Tun AM, Saeed H (2018) Copper deficiency anemia: review article. Ann Hematol 97(9): 1527-1534.

4. Zischka H, Einer C (2018) Mitochondrial copper homeostasis and its derailment in Wilson disease. Int J Biochem Cell Biol 102: 71-75.

5. Bandmann O, Weiss KH, Kaler SG (2015) Wilson's disease and other neurological copper disorders. Lancet Neurol 14(1): 103-113.

6. Ferenci P (2017) Diagnosis of Wilson disease. Handb Clin Neurol 142 171-180.
7. Kelly C, Pericleous M (2018) Wilson disease: more than meets the eye. Postgrad Med J 94(1112): 335-347.

8. Xie JJ, Wu ZY (2017) Wilson's Disease in China. Neurosci Bull 33(3): 323330.

9. Roberts EA, Socha P (2017) Wilson disease in children. Handb Clin Neurol 142: 141-156.

10. Aggarwal A, Bhatt M (2018) Advances in Treatment of Wilson Disease. Tremor Other Hyperkinet Mov (N Y) 8: 525.

11. Członkowska A, Litwin T, Chabik G (2017) Wilson disease: neurologic features. Handb Clin Neurol 142: 101-119.

12. Dzieżyc K, Litwin T, Członkowska A (2017) Other organ involvement and clinical aspects of Wilson disease. Handb Clin Neurol 142: 157-169.

13. Litwin T, Dzieżyc K, Karliński M, Szafrański T, Członkowska A (2016) Psychiatric disturbances as a first clinical symptom of Wilson's disease case report. Psychiatr Pol 50(2): 337-344.

14. Chavez LM, Huang SS, MacDonald I, Lin JG, Lee YC, et al. (2017) Mechanisms of Acupuncture Therapy in Ischemic Stroke Rehabilitation: A Literature Review of Basic Studies. Int J Mol Sci 18(11): 2270.

15. Li ZR, Shen MH, Peng YJ (2005) Progress in researches on the effect of acupuncture in antagonizing oxygen stress. Chin J Integr Med 11(2): 156-160. 